Provided for non-commercial research and education use. Not for reproduction, distribution or commercial use.

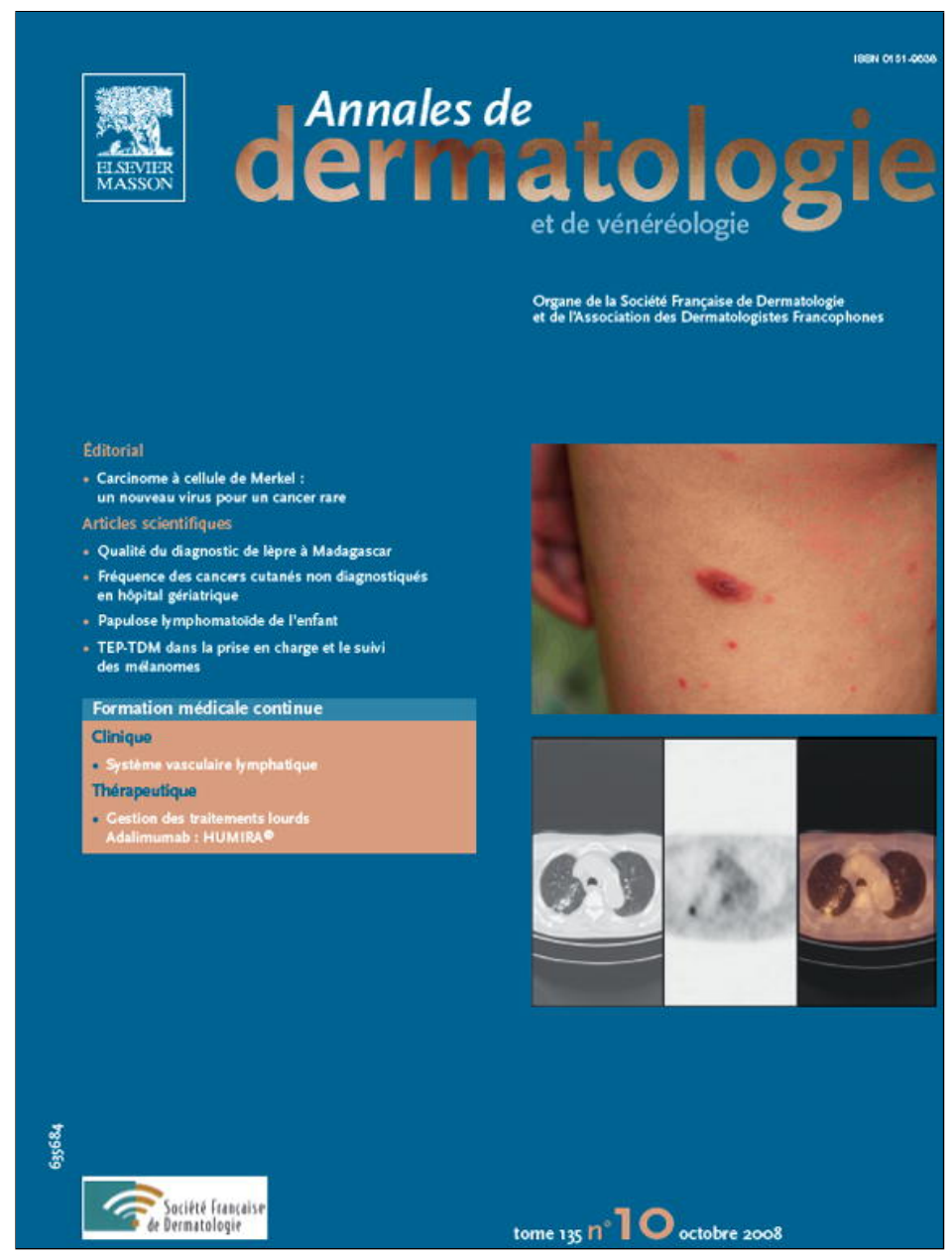

This article appeared in a journal published by Elsevier. The attached copy is furnished to the author for internal non-commercial research and education use, including for instruction at the authors institution and sharing with colleagues.

Other uses, including reproduction and distribution, or selling or licensing copies, or posting to personal, institutional or third party websites are prohibited.

In most cases authors are permitted to post their version of the article (e.g. in Word or Tex form) to their personal website or institutional repository. Authors requiring further information regarding Elsevier's archiving and manuscript policies are encouraged to visit:

http://www.elsevier.com/copyright 


\title{
CLINIQUE
}

\section{Le système vasculaire lymphatique : états des connaissances et implications dermatologiques potentielles}

\section{Lymphatic vasculature: Dermatological implications of emerging concepts}

\author{
A. Henno ${ }^{a, b, c, *}$, C. - M. Lapière ${ }^{b}$, \\ B.V. Nusgens ${ }^{b}, M$. de la Brassinne ${ }^{c}$
}

\author{
a Aspirante FNRS, Belgique \\ ${ }^{\mathrm{b}}$ Laboratoire de biologie des tissus conjonctifs, tour de pathologie B23, centre hospitalier \\ universitaire de Liège, 4000 Liège, Belgique \\ ' Service de dermatologie, centre hospitalier universitaire de Liège B35, domaine \\ universitaire du Sart-Tilman, 4000 Liège, Belgique
}

Reçu le 26 juin 2007 ; accepté le 25 avril 2008

Disponible sur Internet le 3 août 2008

\section{Introduction}

Le système lymphatique possède des fonctions physiologiques diverses telles que l'homéostasie des fluides corporels, l'absorption des graisses alimentaires et la participation aux défenses immunitaires. Si la première description des vaisseaux lymphatiques date du xviie siècle, cette partie du système vasculaire n'a pas fait l'objet d'autant d'attention que le système sanguin. Cela est probablement dû à l'absence de marqueurs spécifiques et au manque de connaissance de sa régulation moléculaire et de sa fonction. Les progrès scientifiques récents ont permis de combler une part de cette lacune, ouvrant la porte à la découverte du rôle pathologique de la dysfonction de l'endothélium vasculaire lymphatique. Les premiers facteurs de croissance et les marqueurs moléculaires spécifiques ont été découverts au milieu des années 1990. Depuis lors, cet endothélium fait l'objet d'une attention

\footnotetext{
* Auteur correspondant.

Adresse e-mail : audrey.henno@student.ulg.ac.be (A. Henno).
}

scientifique croissante. Les liens entre lymphangiogenèse et extension tumorale se précisent, tandis que les bases moléculaires des lymphœdèmes primaires se dévoilent. Un rôle pour l'expansion lymphatique est très probable, mais non encore parfaitement déterminé dans les pathologies inflammatoires. À l'heure de l'évaluation des premiers inhibiteurs de l'angiogenèse en pathologie cancéreuse, nous dressons ici un aperçu des connaissances et espoirs actuels qu'apporte la découverte des systèmes de régulation lymphatique.

\section{Structure du système lymphatique}

Le système lymphatique est un système unidirectionnel débutant par des capillaires à extrémité aveugle qui, dans la peau, prennent naissance au niveau des papilles dermiques. Un réseau lymphatique parallèle au plexus vasculaire dermique superficiel envoie des vaisseaux vers le réseau profond. Les capillaires, généralement plus larges que leurs homologues sanguins, sont dépourvus de valves et de membrane basale continue. Des fibres d'ancrage les lient à la matrice extracellulaire, permettant leur expan- 


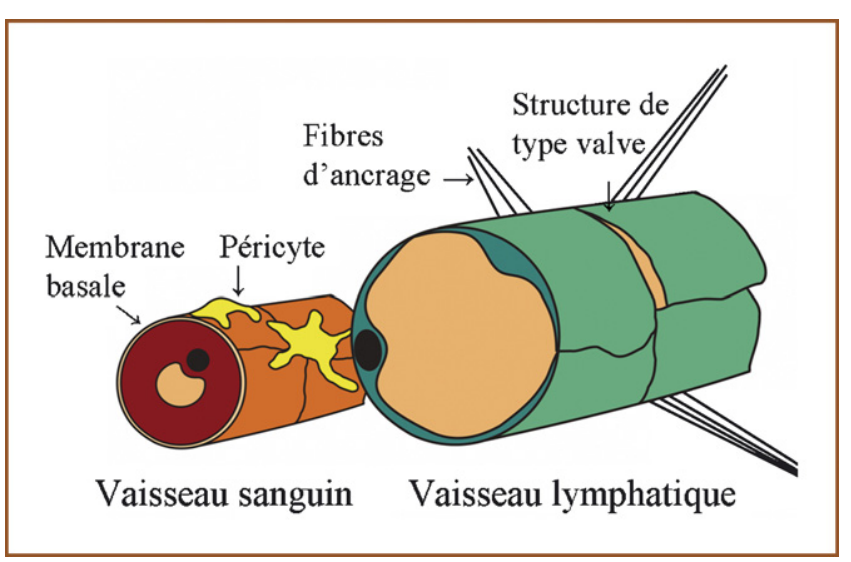

Figure 1. Comparaison schématique d'un capillaire sanguin et d'un capillaire lymphatique. La lumière d'un capillaire lymphatique est généralement plus large et plus irrégulière que celle d'un vaisseau sanguin de même importance. Dans les capillaires lymphatiques, la membrane basale est généralement incomplète et des fibrilles d'ancrage lient le vaisseau aux structures environnantes en déterminant des ouvertures lors des augmentations de pression tissulaire.

sion volumétrique et donc une résorption accrue de liquide extracellulaire en cas d'hyperpression tissulaire (Fig. 1) [1]. Les vaisseaux collecteurs (que l'on retrouve dans l'hypoderme) sont en revanche munis de valves et entourés d'une membrane basale. Ils accompagnent fréquemment les vaisseaux sanguins et confluent progressivement jusqu'au canal thoracique qui débouche dans la veine sous-clavière.

\section{Embryologie de la lymphangiogenèse}

La formation des vaisseaux lymphatiques survient après celle du réseau vasculaire sanguin. Les éléments récents de la recherche fondamentale semblent corroborer la théorie énoncée par Sabin au début du xx ${ }^{\mathrm{e}}$. Il semble en effet que les cellules endothéliales lymphatiques naissent par bourgeonnement à partir des veines embryonnaires des zones jugulaires et périmésonéphriques [2]. Elles migrent pour former les sacs et le plexus lymphatique primaire composé de vaisseaux capillaires dans l'embryon de six à sept semaines (Fig. 2).

Deux éléments sont essentiels à ce processus : le système ligand-récepteur formé par le vascular endothelial growth factor C (VEGF-C) et le vascular endothelial growth factor receptor 3 (VEGFR3) et le facteur de transcription prosperohoméobox-1 (prox-1).

Le VEGF-C est un facteur de survie et un agent chimiotactique essentiel durant la lymphangiogenèse embryonnaire. Les expériences chez la souris démontrent en effet que sa délétion entraîne l'absence complète de vaisseaux lymphatiques et est létale durant le développement embryonnaire. On note alors l'absence de migration des cellules destinées à un devenir lymphatique, ces altérations étant corrigées par l'administration exogène de VEGF-C ou -D. Lorsque la délétion est hétérozygote, seuls une hypoplasie lymphatique cutanée sévère et un lymphœdème sont observés [3]. À l'inverse, une surexpression de ce facteur de croissance par l'épiderme entraîne l'hyperplasie des vaisseaux lymphatiques [4].
Le VEGFR3 présent sur les cellules endothéliales lymphatiques permet la signalisation par VEGF-C et -D. Ce récepteur est exprimé par toutes les cellules endothéliales vasculaires au début du développement embryonnaire avant que son expression ne se voit restreinte aux cellules endothéliales lymphatiques, avec quelques exceptions pour l'endothélium vasculaire sanguin de certaines glandes endocrines et d'organes tels que la moelle épinière [5]. Plusieurs conditions pathologiques, comme le cancer, sont accompagnées d'une réexpression de ce facteur sur l'endothélium vasculaire [6]. Chez la souris, une délétion totale de ce récepteur entraîne des anomalies dans le remodelage des vaisseaux sanguins et une létalité embryonnaire. Des mutations dans le domaine tyrosine kinase de ce récepteur ont été découvertes chez des patients atteints de la maladie de Milroy [7]. Le VEGFR3 est en fait nécessaire à la mise en place initiale du système cardiovasculaire, puis du système lymphatique [2].

Notons que le VEGF-D, un autre facteur de croissance de la famille du VEGF reconnu par le VEGFR3 et le VEGFR2, est mitogène pour les cellules endothéliales et pro-angiogène. Son expression est abondante dans la peau et les poumons durant l'embryogenèse puis se réduit. Les souris déficientes n'ont cependant pas de phénotype vasculaire lymphatique spécifique [8].

Prospero-homéobox-1 (prox-1) est un gène homéobox nécessaire au développement lymphatique. Il induit le phénotype «lymphatique» dans les cellules endothéliales veineuses et sa délétion s'accompagne d'une absence complète de vaisseaux lymphatiques. Sa surexpression dans les cellules endothéliales vasculaires humaines entraîne la suppression de plusieurs gènes spécifiques des cellules endothéliales des vaisseaux sanguins et une activation de la transcription de gènes spécifiques des cellules endothéliales lymphatiques [2].

$\mathrm{Au}$ cours de l'embryogenèse, une partie des cellules des veines jugulaires expriment prox-1. Des cellules mésenchymateuses environnantes produisent le VEGF-C. Alors que toutes les cellules endothéliales embryonnaires présentent le VEGFR3, seules celles qui expriment prox-1 possèdent la capacité de migrer dans le gradient de VEGF-C. L'assemblage en tubules des cellules permet ensuite la formation des premiers sacs lymphatiques [9] (Fig. 2). Les ganglions lymphatiques se développent à partir des sacs lymphatiques primitifs par protrusion du tissu conjonctif et le développement des organes lymphoïdes secondaires se fait de manière séquentielle, régulé par des gènes distincts de ceux qui contrôlent la lymphangiogenèse [2].

\section{Lymphangiogenèse chez l'adulte: facteurs de croissance et récepteurs nouvellement identifiés}

À l'image du système vasculaire sanguin, le réseau lymphatique adulte est physiologiquement dans un état stable et l'activation de la lymphangiogenèse s'observe dans certaines conditions particulières comme la réparation des plaies ou se rencontre de manière excessive dans des conditions pathologiques comme les cancers. Les facteurs de croissance précédemment décrits (VEGF-C et VEGF-D) 


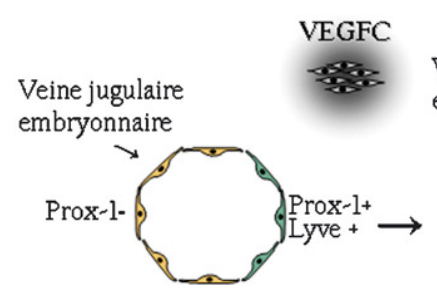

Engagement vers le phénotype lymphatique

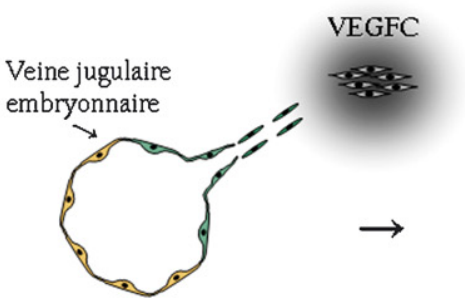

Bourgeonnement et migration des cellules endothéliales lymphatiques

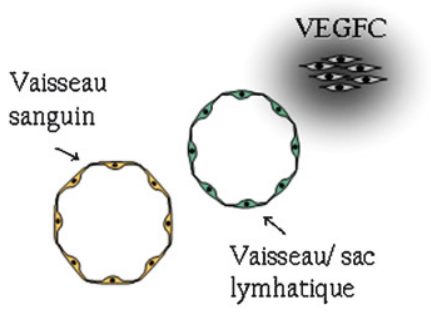

Formation des sacs lymphatiques et lymphangiogenèse

Figure 2. Le système lymphatique est d'origine veineuse. Comme décrit par Saharinen et al. [9], une sous-population de cellules endothéliales exprime le facteur prox-1 de manière polarisée dans la veine jugulaire au jour embryonnaire dix chez la souris et aux environs de la sixième ou septième semaine embryonnaire chez l'homme. Ces cellules surexpriment d'autres marqueurs et gènes lymphatiques tandis qu'elles sous-expriment des gènes vasculaires. Elles sont en outre capables de migrer vers le gradient de VEGF-C produit par des cellules mésenchymateuses environnantes pour former les sacs lymphatiques primitifs. Le système vasculaire lymphatique se forme à partir de ceux-ci de manière centrifuge.

interviennent ici aussi, en collaboration avec le récepteur VEGFR3 [9].

\section{Marqueurs moléculaires des vaisseaux lymphatiques}

Depuis quelques années, des anticorps spécifiques permettent le marquage immunohistochimique des vaisseaux lymphatiques. Ceux-ci sont dirigés contre le VEGFR3, la podoplanine (protéine de surface des cellules endothéliales lymphatiques ayant un rôle dans leur adhésion, leur migration et la formation de tubes), le facteur prox-1 ou Lyve-1 (récepteur de l'acide hyaluronique spécifique de l'endothélium lymphatique) $[9,10]$.

\section{Réparation des plaies}

Durant la cicatrisation, la régénération des vaisseaux lymphatiques survient après celle des vaisseaux sanguins [1]. Cette régénération ne se ferait pas par bourgeonnement à partir des vaisseaux préexistants, mais par un processus de migration et d'organisation unidirectionnelle de cellules individuelles dans la direction du flux de fluide interstitiel (de la partie distale vers la partie proximale). Ces cellules se rejoignent pour former des structures tubulaires. Le flux du fluide interstitiel est nécessaire à ce processus. Les taux de VEGFC sont élevés lors des premières étapes de ce processus mais se réduisent pendant les phases d'organisation et de maturation des lymphatiques, suggérant un rôle initiateur pour le VEGFC [11].

\section{Le lymphœdème primaire: fondements génétiques et espoirs thérapeutiques}

Le lymphœdème est une pathologie chronique et invalidante définie comme le gonflement d'une partie du corps dû à l'accumulation de fluide interstitiel riche en protéines secondairement à une malformation ou une dysfonction du système lymphatique. On distingue les lymphœdèmes primaires et secondaires. Les premiers sont liés à une anomalie primitive des vaisseaux lymphatiques et les seconds à leur destruction ou leur obstruction. Les principales causes de lymphœdème secondaires sont les infections, les tumeurs, les interventions chirurgicales et la radiothérapie [12]. Les lymphœdèmes primaires sont classés en congénitaux, précoces et tardifs en fonction de leur âge d'apparition (avant un an, entre un et 35 ans et après 35 ans, respectivement). Parmi ces lymphœdèmes primaires, on retrouve les formes familiales qui comprennent la maladie de Milroy (lymphœdème primaire congénital), la maladie de Meige ou encore le lymphœdème-distichiasis (précoces) [13].

La maladie de Milroy débute à la naissance et s'accompagne d'une hypoplasie des vaisseaux lymphatiques responsable d'une hypertrophie globale d'un ou plusieurs membres. Récemment, des auteurs ont mis en évidence diverses mutations chez des patients hétérozygotes, provoquant l'expression d'un récepteur VEGFR3 au domaine tyrosine kinase inactif $(5 q 34-5 q 35)$. Cette altération empêche la transmission du signal de ce récepteur et, de plus, réduit sa dégradation, entraînant alors une accumulation du VEGFR3 pathologique et inefficace à la surface cellulaire [14]. Un modèle murin présentant des mutations du domaine tyrosine kinase du VEGFR3 a également été décrit (souris Chy) [15]. Un lymphœdème des pattes et une ascite chyleuse avec absence de lymphatiques sous-cutanés sont observés dans ce modèle.

Le lymphœdème-distichiasis, survient à la puberté. II associe le distichiasis (double rangée de cils) au lymphœdème, et parfois à des troubles divers tels que photophobie ou ectropion mais aussi à des malformations cardiaques, fentes palatines ou kystes spinaux. La lymphangiographie objective un nombre de vaisseaux lymphatiques à la limite supérieure de la normale et leur dilatation modérée au niveau dermique ainsi que l'épaississement de leurs parois, avec présence de cellules musculaires lisses ectopiques à leur pourtour. Les valves lymphatiques de ces patients 
sont défectueuses de même que les valves veineuses, qui peuvent être incompétentes. Une mutation du gène FOXC2 (16q24), facteur de transcription responsable de la formation des valves et de l'inhibition du recrutement des cellules musculaires lisses ou des péricytes autour des vaisseaux lymphatiques, a été mise en évidence dans cette pathologie. Des manipulations génétiques chez la souris ont confirmé l'effet de cette mutation sur le phénotype [16].

Le lymphœdème n'est pas une pathologie dénuée de complications (infections, susceptibilité tumorale) en partie expliquées par le fait qu'il s'agit d'un site immunologiquement vulnérable [17]. Les traitements actuels de l'insuffisance vasculaire lymphatique (traitements physiques empêchant le gonflement tissulaire, chirurgie de résection ou de reconstruction et médicaments de type diurétiques, benzopyrones, coumariniques et flavonoïdes) sont parfois lourds et insuffisants. Un espoir thérapeutique réside cependant dans la thérapie génique (ou protéique) par le VEGF-C, du moins chez les patients exempts de cancers, comme nous le verrons au paragraphe suivant. L'administration de VEGF-C induit en effet la croissance de vaisseaux lymphatiques au niveau cutané de la souris Chy [15], comme dans d'autres modèles animaux reproduisant les lymphœdèmes secondaires [18]. Actuellement, le VEGF-C est l'outil moléculaire le plus prometteur pour le traitement des lymphœdèmes primaires et secondaires, bien qu'efficacité et sécurité à long terme restent à prouver.

\section{Lymphangiogenèse tumorale, métastases et cancers cutanés}

La plupart des cancers peuvent s'accompagner d'une dissémination métastatique par voie hématogène ou lymphatique. L'envahissement ganglionnaire constitue un facteur pronostique important. La stimulation de la lymphangiogenèse par les facteurs de croissance accroît les métastases lymphatiques. Plusieurs études ont mis en évidence une corrélation entre l'expression de VEGF-C ou VEGF-D et l'invasion vasculaire, lymphatique ou ganglionnaire [2]. Il existe une corrélation entre l'expression de ces facteurs de croissance par la tumeur, les métastases à distance et un mauvais pronostic clinique $[19,20]$. Chez la souris, la surexpression de VEGF-C ou VEGF-D provoque la lymphangiogenèse tumorale et accroît les métastases lymphatiques [21].

Certains cancers cutanés tels le mélanome et les carcinomes de la tête et du cou contiennent un nombre accru de vaisseaux lymphatiques intratumoraux et péritumoraux. Ces derniers pourraient être rendus responsables de la dissémination métastatique, répondant à la production de facteurs de croissance par les cellules tumorales en formant des bourgeons à lumière large permettant un accès aisé aux cellules malignes. À l'opposé, les vaisseaux intratumoraux pourraient être en collapsus. Cette opinion est cependant controversée car certaines études montrent une corrélation entre un nombre accru de vaisseaux lymphatiques intratumoraux dans les carcinomes de la tête et du cou et un mauvais pronostic clinique [20]. Notons que l'endothélium lymphatique peut participer à l'invasion tissulaire par la sécrétion de chimiokines telles que le CCL21 dont le ligand CCR7, normalement trouvé à la surface des neutrophiles et des macrophages, est parfois exprimé par les cellules cancéreuses. Les vaisseaux collecteurs qui drainent la région tumorale se dilatent généralement sous l'effet du VEGF-C et facilitent encore le transit de groupes de cellules métastatiques [2].

Divers travaux portant sur le mélanome cutané décrivent la lymphangiogenèse tumorale comme le marqueur pronostique le plus sensible de l'invasion du ganglion sentinelle, avant même l'épaisseur tumorale [19]. Cela reste cependant controversé [22].

Des protéines de fusion entre la partie constante d'une immunoglobuline et le VEGFR3 ou des anticorps qui peuvent fixer les deux facteurs VEGF-C et VEGF-D permettent de bloquer les métastases lymphatiques dans certains modèles animaux. L'inhibition du système VEGFR3-VEGF-C et- D chez l'homme est un espoir dans le blocage des métastases [2].

\section{La maladie de Kaposi}

Quatre formes épidémiologiques de maladies de Kaposi sont distinguées:

- la forme classique (survenant chez des patients de type méditerranéen ou d'ascendance juive et âgés de plus de 50 ans) ;

- la forme endémique (survenant chez des patients d'âge variable dans certaines régions d'Afrique équatoriale, surtout la région des grands lacs);

- la forme associée aux immunodépressions acquises iatrogènes, notamment après transplantation rénale;

- la forme associée à l'infection par le virus du Sida (VIH).

Les hommes sont plus fréquemment touchés dans toutes ces formes [23]. Cette pathologie affecte surtout la peau et se manifeste par des lésions de type papule, nodule ou plaque. La muqueuse buccale n'est pas épargnée. L'histologie comprend une prolifération de cellules fusiformes, une angiogenèse importante avec formation de fentes vasculaires et une inflammation. La définition de la maladie de Kaposi est rendue difficile par la présence de multiples types cellulaires (cellules endothéliales, fibroblastes, macrophages, dendrocytes...), l'absence de monoclonalité et d'anomalie chromosomique décelable. Les cultures cellulaires réalisées à partir des lésions ne survivent qu'à un nombre limité de passages et les cellules isolées produisent des lésions bénignes et transitoires chez les souris nues [24]. La prolifération observée dans cette pathologie ne serait pas néoplasique et pourrait être une réponse hyperplasique à divers stimuli environnementaux [23].

Les séquences $A D N$ du virus de l'herpès humain (HHV8) ont été découvertes dans l'ADN des lésions de maladie de Kaposi et cette présence a été associée à toutes les formes de la pathologie [24], indiquant une étiologie commune pour celles-ci en dépit de différences épidémiologiques. Le virus peut être décelé avant le développement de la maladie, ce qui renforce l'hypothèse d'un rôle étiologique nécessaire [24]. Il est en outre associé à la maladie de Castleman multicentrique de type plasmocytaire et aux lymphomes primitifs 
des séreuses [25]. Le génome de HHV8 a été séquencé et de nombreux gènes viraux semblent nécessaires pour la régulation de la prolifération cellulaire, l'inhibition de l'apoptose et la réponse immunitaire [25]. Il contient en effet des homologies de séquence avec des cyclines cellulaires de type $D$ pouvant favoriser le passage de la phase $G 1$ à la phase $S$. Il code pour un homologue du récepteur couplé à la protéine-G qui est responsable de l'activation du facteur de transcription AP-1 impliqué dans la prolifération cellulaire et pour un gène homologue au $b c l-2$ cellulaire capable d'inhiber l'apoptose. La protéine de latence nucléaire exprimée dans $90 \%$ des cellules fusiformes constituant l'infiltrat cellulaire de Kaposi nodulaire, mais aussi dans les lésions initiales, est essentielle au maintien de HHV8 sous forme épisomal [25].

Les cellules fusiformes de la maladie de Kaposi expriment à la fois des marqueurs vasculaires sanguins (CD34, collagène de type IV) et lymphatiques (VEGFR3, podoplanine, prox-1, Lyve-1), suggérant leur origine endothéliale. Elles sont marquées par l'anticorps D2-40 spécifique des cellules endothéliales lymphatiques. Le profil transcriptomique des cellules de la maladie de Kaposi nodulaire analysé par la technique des micro arrays (ou des micropuces à ADN) se rapproche plus de celui des cellules endothéliales que de celui des autres types cellulaires et plus encore des cellules endothéliales lymphatiques que des cellules sanguines [26]. La signature de la maladie de Kaposi (la différence entre les gènes exprimés dans cette maladie et les gènes exprimés au même niveau dans la peau normale) est constituée de 1482 gènes dont les marqueurs lymphatiques VEGFR3, podoplanine et le CD206 (un récepteur du mannose) et des marqueurs sanguins. Cela suggère que les cellules fusiformes ne représentent pas un seul type cellulaire, ce qui pourrait être une conséquence de l'infection à HHV8 [26].

Les cellules les plus susceptibles à l'infection par le HHV8 in vitro sont les cellules endothéliales et parmi celles-ci, les cellules lymphatiques présentent un plus grand nombre de copies de son génome que les cellules sanguines [26]. L'infection des cellules endothéliales vasculaires sanguines par le HHV8 induit l'expression de plusieurs gènes spécifiques de l'endothélium lymphatique dont prox-1, Lyve-1 et la desmoplakine [27] et donc un profil génétique plus proche des cellules lymphatiques tandis que l'expression de gènes spécifiques des cellules sanguines est réduite (laminine, CD44, ICAM-1). L'inverse est également vrai car l'expression de gènes spécifiques des cellules endothéliales lymphatiques, dont prox-1, est réduite après leur infection par le virus tandis que celle de gènes sanguins est accrue. Ces résultats suggèrent que l'infection à HHV8 induit une reprogrammation transcriptionnelle des cellules sanguines vers un phénotype lymphatique et vice versa. Ce virus serait le premier pathogène humain capable d'une telle reprogrammation transcriptomique des cellules endothéliales [26]. La protéine de latence virale serait impliquée dans l'induction de prox-1 dans les cellules sanguines infectées, ce dernier facteur jouant un rôle important dans le processus de reprogrammation lymphatique des cellules sanguines. D'autres facteurs de transcription semblent cependant également impliqués car l'inhibition de l'activité de prox-1 n'inhibe que partiellement l'expression de marqueurs lymphatiques [27].

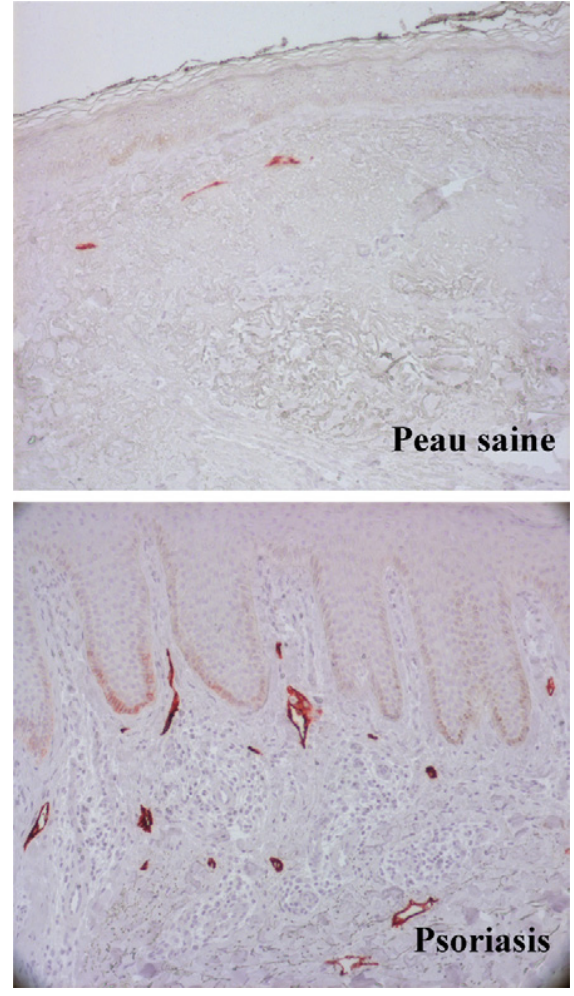

Figure 3. Immunohistochimie représentant les vaisseaux lymphatiques cutanés (marqués en rouge) dans la peau saine et dans le psoriasis. Dans la peau saine, un réseau capillaire parallèle au plexus vasculaire dermique superficiel envoie des vaisseaux vers le réseau profond. Dans le psoriasis, on note un accroissement du nombre de capillaires lymphatiques du derme superficiel et leur dilatation.

\section{Pathologies inflammatoires}

L'inflammation est accompagnée d'une prolifération des vaisseaux lymphatiques qui participent à la réponse inflammatoire par leur implication dans le transport des lymphocytes vers les ganglions lymphatiques. Les macrophages activés sont capables d'exprimer le VEGF-C et -D. Dans le rejet des greffes rénales, l'infiltrat inflammatoire est parsemé de capillaires lymphatiques en prolifération. De même, les infections respiratoires de la souris par les mycoplasmes sont accompagnées d'une lymphangiogenèse médiée par le VEGF-C et -D. La synoviale des patients atteints de polyarthrite rhumatoïde exprime le VEGF-C [2]. Dans la peau, les vaisseaux lymphatiques jouent un rôle dans le contrôle de l'œdème et de l'inflammation secondaires à l'exposition aux UVB [28]. Par ailleurs, le psoriasis s'accompagne d'une expansion lymphatique dont le rôle est encore mal connu (Fig. 3) [29].

\section{Conclusion}

Les connaissances récemment acquises dans la régulation de la lymphangiogenèse ont permis d'identifier diverses molécules permettant de spécifier la cellule endothéliale lymphatique et capables de stimuler ou inhiber la lymphangiogenèse. Ces observations ouvrent des perspectives thérapeutiques génétiques et biologiques innovantes tant 
pour le lymphœdème qu'en ce qui concerne l'extension métastatique en cancérologie. La maladie de Kaposi pourrait en outre bénéficier de l'apport de thérapeutiques inhibant la lymphangiogenèse. La poursuite des recherches dans ce domaine est déterminante afin de définir les interactions complexes de ce système avec son correspondant sanguin ainsi que les mécanismes moléculaires précis responsables des pathologies discutées dans cette revue.

\section{Références}

[1] Hong YK, Lange-Asschenfeldt $B$, Velasco P, Hirakawa S, Kunstfeld R, Brown LF, et al. VEGF-A promotes tissue-repairassociated lymphatic vessel formation via VEGFR2 and the $\alpha 1 \beta 1$ and $\alpha 2 \beta 1$ integrins. FASEB $J$ 2004;18:1111-3.

[2] Alitalo K, Tammela T, Petrova TV. Lymphangiogenesis in development and human disease. Nature 2005;438:946-53.

[3] Karkkainen MJ, Haiko P, Sainio K, Partanen J, Taipale J, Petrova TV, et al. Vascular endothelial growth factor $C$ is required for sprouting of the first lymphatic vessels from embryonic veins. Nat Immunol 2004;5:74-80.

[4] Jeltsch M, Kaipainen A, Joukov V, Meng X, Lakso M, Rauvala $\mathrm{H}$, et al. Hyperplasia of lymphatic vessels in VEGF-C transgenic mice. Science 1997;276:1423-5. Erratum in Science 1997, 277:463.

[5] Partanen TA, Arola J, Saaristo A, Jussila L, Ora A, Miettinen M, et al. VEGF-C and VEGF-D expression in neuroendocrine cells and their receptor, VEGFR-3, in fenestrated blood vessels in human tissues. FASEB J 2000;14:2087-96.

[6] Kubo H, Fujiwara T, Jussila L, Hashi H, Ogawa M, Shimizu K, et al. Involvement of vascular endothelial growth factor receptor3 in maintenance of integrity of endothelial cell lining during tumor angiogenesis. Blood 2000;96:546-53.

[7] Saaristo A, Karkkainen M, Alitalo K. Insights into the molecular pathogenesis and targeted treatment of lymphoedema. Ann $\mathrm{N}$ Y Acad Sci 2002;979:94-110.

[8] Baldwin M, Halford M, Roufail S, Williams R, Hibbs M, Grail $D$, et al. Vascular endothelial growth factor-D is dispensable for development of the lymphatic system. Mol Cell Biol 2005;25:2441-9.

[9] Saharinen P, Tammela T, Karkkainen M, Alitalo K. Lymphatic vasculature: development, molecular regulation and role in tumor metastasis and inflammation. Trends Immunol 2004;25:387-95.

[10] Banerji S, Ni J, Wang S, Clasper S, Su J, Tammi R, et al. LYVE-1, a new homologue of the CD44 glycoprotein, is a lymph-specific receptor for hyaluronan. J Cell Biol 1999;144:789-801.

[11] Rutkowski JM, Boardman KC, Swartz MA. Characterization of lymphangiogenesis in a model of adult skin regeneration. Am J Physiol Heart Circ Physiol 2006;291:H1402-10.

[12] Lazareth I. Classification des lymphœdèmes. Rev Med Interne $2002 ; 23: 375 s-8 s$.
[13] Rockson S. Lymphoedema. Am J Med 2001;110:288-95.

[14] Karkkainen M, Jussila L, Ferrell R, Finegold D, Alitalo K. Molecular regulation of lymphangiogenesis and targets for tissue oedema. Trends Mol Med 2001;7:18-22.

[15] Karkkainen MJ, Saaristo A, Jussila L, Karila KA, Lawrence EC, Pajusola K, et al. A model for gene therapy of human hereditary lymphedema. Proc Natl Acad Sci U S A 2001;98:12677-82.

[16] Petrova TV, Karpanen T, Norrmen C, Mellor R, Tamakoshi T, Finegold D, et al. Defective valves and abnormal mural cell recruitment underlie lymphatic vascular failure in lymphoedema distichiasis. Nat Med 2004;10:974-81.

[17] Ruocco V, Schwartz R, Ruocco E. Lymphedema: an immunologically vulnerable site for development of neoplasms. J Am Acad Dermatol 2002;47:124-7.

[18] Szuba A, Skobe M, Karkkainen M, Shin W, Beynet D, Rockson N, et al. Therapeutic lymphangiogenesis with human recombinant VEGF-C. FASEB J 2002;16:1985-7.

[19] Dadras SS, Lange-Asschenfeld B, Velasco P, Nguyen L, Vora A, Muzikansky A, et al. Tumor lymphangiogenesis predicts melanoma metastasis to sentinel lymph nodes. Mod Pathol 2005; 18:1232-42.

[20] Maula SM, Luukkaa M, Grenman R, Jackson D, Jalkanen S, Ristamaki R. Intratumoral lymphatics are essential for the metastatic spread and prognosis in squamous cell carcinomas of the head and neck region. Cancer Res 2003;63:1920-6.

[21] Mandriota SJ, Jussila L, Jeltsch M, Compagni A, Baetens $\mathrm{D}$, Prevo R, et al. Vascular endothelial growth factor-Cmediated lymphangiogenesis promotes tumour metastasis. EMBO J 2001;20:672-82.

[22] Straume 0, Akslen LA. Lymphatic vessel density and prognosis in cutaneous melanoma. Br J Cancer 2004;91:1224-5.

[23] Gallo R. The enigma of Kaposi's sarcoma. Science 1998;282:1837-9.

[24] Reitz M, Nerurkar L, Gallo R. Perspective on Kaposi's sarcoma: facts, concepts and conjectures. J Natl Cancer Inst 1999;91:1453-8.

[25] Dupin N, Calvez V. Virus HHV-8/KSHV. I- Aspects épidémiologiques et moléculaires. Ann Dermatol Venereol 2000;127:528-31.

[26] Wang $H$, Trotter $M$, Lagos $D$, Bourboulia D, Henderson S, Mäkinen T, et al. Kaposi sarcoma herpesvirus-induced cellular reprogramming contributes to the lymphatic endothelial gene expression in Kaposi sarcoma. Nat Genet 2004;36:687-93.

[27] Hong Y, Foreman K, Shin J, Hirakawa S, Curry C, Sage D, et al. Lymphatic reprogramming of blood vascular endothelium by Kaposi sarcoma-associated herpesvirus. Nat Genet 2004;36:683-5.

[28] Kajiya K, Detmar M. An important role of lymphatic vessels in the control of UVB-induced edema formation and inflammation. J Invest Dermatol 2006;126:919-21.

[29] Henno A, Lapière CM, de la Brassinne $M$, Nusgens $B$. The expression of angiogenesis and lymphangiogenesis markers is higher in normal skin of psoriatic patients than in healthy volunteer skin. J Invest Dermatol 2006;126:S3-99 [abstract]. 